\title{
Using Object Affordances to Improve Object Recognition
}

\author{
C. Castellini, T. Tommasi, N. Noceti, F. Odone, and B. Caputo
}

\begin{abstract}
The problem of object recognition has not yet been solved in its general form. The most successful approach to it so far relies on object models obtained by training a statistical method on visual features obtained from camera images. The images must necessarily come from huge visual datasets, in order to circumvent all problems related to changing illumination, point of view, etc. We hereby propose to also consider, in an object model, a simple model of how a human being would grasp that object (its affordance). This knowledge is represented as a function mapping visual features of an object to the kinematic features of a hand while grasping it. The function is practically enforced via regression on a human grasping database. After describing the database (which is publicly available) and the proposed method, we experimentally evaluate it, showing that a standard object classifier working on both sets of features (visual and motor) has a significantly better recognition rate than that of a visual-only classifier.
\end{abstract}

Index Terms-Biologically inspired feature extraction, learning systems, robot tactile systems, robot vision systems.

\section{INTRODUCTION}

$\mathbf{C}$ ONSIDER the objects in Fig. 1. How do we know that they are all cups? The answer is intuitive: they can all be used to contain liquids and to drink, and have actually been designed to this end. Although very little in their visual appearance ties them together, a human being will immediately know what can be done with such objects since he has done it at some time in the past. As a matter of fact, the category of an object is often determined more by its function rather than by its visual appearance; this idea has led Gibson in the 1970s [1], [2] to define objects in terms of their affordances - "what can be done with them." It is probably this key intuition that makes human object recognition so robust.

This idea, we believe, could be profitably used to solve the general problem of mechanical object recognition. Consider the above cups: traditionally, an object recognition system would be trained on a very large database of images of very diverse cups, shot in different conditions of illumination, from different points of view, etc. This is clearly incomplete and resource-con-

Manuscript received February 23, 2010; revised October 14, 2010; accepted December 15, 2010. Date of publication January 24, 2011; date of current version September 14, 2011. This work was supported by the EU Projects DIRAC (IST-027787, BC, and TT) and Contact (NEST 5010, CC).

C. Castellini was with the LIRA-Lab, Università degli Studi di Genova, Genova, Italy. He is now with the DLR, German Aerospace Research Center, Oberpfaffenhofen, Germany (e-mail claudio.castellini@dlr.de).

T. Tommasi and B. Caputo are with the Idiap Research Institute, CH-1920 Martigny, Switzerland (e-mail bcaputo@idiap.ch; ttommasi@idiap.ch).

N. Noceti and F. Odone are with DISI, Università degli Studi di Genova, Genova, Italy (e-mail odone@disi.unige.it; noceti@disi.unige.it).

Digital Object Identifier 10.1109/TAMD.2011.2106782 suming [3]. But what if the system had an idea of how to grasp something which looks like a cup? In that case a new, strong "semantic" element could be used to tie together the three objects above in the category of cups. The use of object affordances to improve the classic solution to object recognition, in which visual features only are exploited, has actually been circulating for a while but it is still unclear how to mechanically enforce it. We hereby propose the use of grasping motor data (i.e., kinematic data obtained from human hands while grasping) to encode the affordances of an object, and then to use the representation of object affordances to improve object recognition.

To test this idea, a total number of 5200 human grasping sequences have been recorded from 20 subjects. Each sequence consists of the video and kinematic recording of the act of grasping one of five objects with one of seven grasping shapes, chosen from standard grasping taxonomies such as Cutkosky's [4]. (Recording of the hand kinematics is done via a sensorized glove and a magnetic tracker.) These sequences are collected in the CONTACT visuo-motor grasping database (VMGdB), presented and described in this very paper. ${ }^{1}$ Using this database and a simple regression schema based upon artificial neural networks, we then build a function, called visuo-motor map (VMM), mapping visual features of an object to an associated grasp. Since in general many different grasps are associated with the same objects, the VMM is here associating an "average" grasp posture to each object, which is not guaranteed to correspond to a physically feasible grasp but still is deemed to carry enough information on the affordances of that object.

At this point, to test the effectiveness of the idea, a standard classifier (namely a support vector machine) is used to classify the objects in the database using either: 1) the visual features only, as is standard in object recognition; 2) the motor features only as recorded by the sensorized glove; 3 ) a combination of these features sets; and 4) a combination of the visual features and the motor features as reconstructed by the VMM. The latter scenario is of course more realistic since in most real-life applications (and in real life as well) the only available input is visual. The hope is that the augmented object classifiers perform dramatically better than the standard one when the real motor features are added; and significantly better when the reconstructed ones are used. Our experimental results confirm this hypothesis, even given the simplifying assumptions made in this work.

The paper is organized like this: after an overview of related work, in Section II, we describe the VMGdB. Section III defines the framework; we then show the experimental results (Section IV) and draw conclusions in Section V.

\footnotetext{
${ }^{1}$ The VMGdB is available at the following URI: http://slipguru.disi.unige.it/ Research/VMGdB for download.
} 

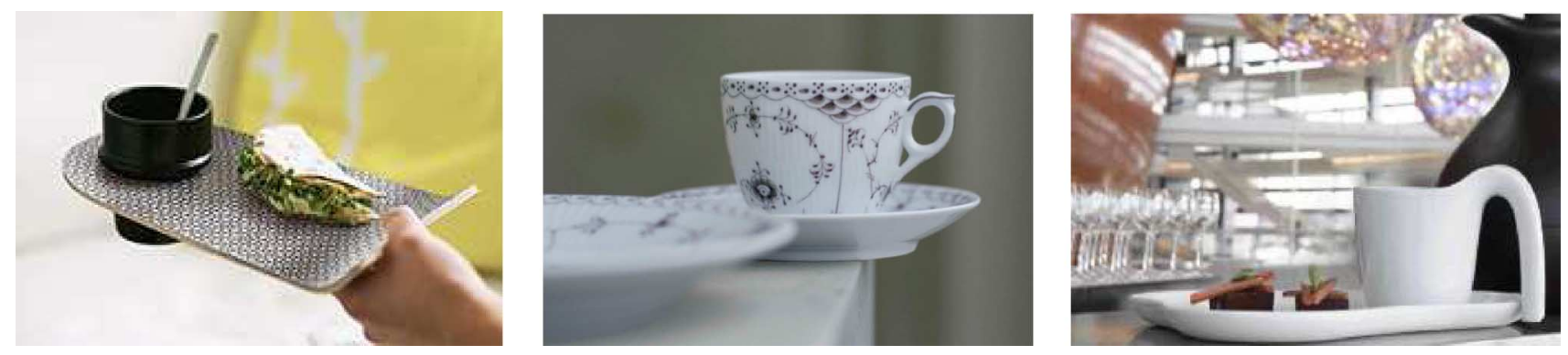

Fig. 1. Three very different cups: (left) the Pick Up mug by Höganäs (2009); (center) the Black Flute Half Lace coffee cup (1775); and (right) the 'Ole mug (1997), both by Royal Copenhagen.

\section{A. Related Work}

The capability to recognize and categorize objects is a crucial ability for an autonomous agent; and in robotics, it is inextricably woven with the ability of grasping an object. In cognitive science, the theoretical link between vision and manipulation was provided by Gibson, according to whom an object is characterized by three properties: 1) it has a certain minimal and maximal size related to the body of an agent; 2) it shows temporal stability; and 3) it is manipulable by the agent. These properties imply that the object is defined in relation to an embodied agent able to manipulate the object. Therefore, the set of possible manipulation actions are a crucial part of the object definition itself.

Interestingly, the theory of affordances has recently found neurological evidence, it is claimed, in the mirror neurons paradigm [5], [6]. According to it, structures exist in the high primates' brain which will fire if, and only if, an object is grasped (which mainly involves the sensorimotor system) or is seen grasped by an external agent (involving the visual system only, [7]). In addition to the original findings in monkeys, very recent evidence has been produced for the existence of such structures in humans [8]. If this is true, then the human object classification is so robust exactly because we know what to do with the objects we see-a capability which machines lack, so far.

This idea has so far been little exploited; among the positive cases there are [9] and [10] who take an exquisitely robotic perspective, letting their systems acquire motor information about objects by having a humanoid robot manipulating them. Our work draws inspiration from [9] and it represents an extension and a further exploration of its topic. On the other hand, the vast majority of work on object recognition and categorization models objects starting from static images, without taking into account their 3-D structure and their manipulability [11], [3]. An interesting exception is [12] where and-or trees and 3-D features are used to categorize objects according to how well they fit a functional profile.

Few very recent attempts try to capture the Gibson's view. The approach proposed in [13] presents a Bayesian framework that unifies the inference processes involved in object categorization and localization, action understanding and perception of object reaction. The joint recognition of objects and actions is based on shape and motion, and the models take as input video data. In [14], Kyellstrom et al. consider objects as contextual information for recognizing manipulation actions and vice versa.
The action-object dependence is modeled with a factorial conditional random field with a hierarchical structure. In both approaches, objects and their affordances are first modeled separately, and combined together in a second step. This does not consider the embodiment of the agent manipulating the objects.

\section{The DATABASE}

The CONTACT VMGdB is the result of recording the visual and kinematic content of grasping acts made by several human subjects, in changing conditions of illumination.

A) Experimental Protocol: The subjects (all right-handed) would sit comfortably on a chair in front of a desk. Their right arm and hand would be resting on the arm of the chair. An object would be placed in a predefined position onto the desk. Then, the subject would be instructed to: 1) reach for and grasp the object with his/her right hand (the grasping instant being signalled by a beep);2) drop it somewhere else in the workspace (the releasing instant being signalled by another, different beep); 3) put the right arm and hand back in the resting position; and 4) put the object back in the original position with the left arm and hand. The desk was uniformly dark green and nonreflective; the objects were chosen to be colorful; the illumination was provided by two windows looming over the desk. Intentionally we did not fix the illumination, which changed over time, since acquisition sessions spanned over a week, in the morning, afternoon and evening. Before each experiment we would fix the white balance of the cameras in order to avoid saturation. Fig. 2 shows a typical reach-and-grasp sequence, as seen by the two cameras.

B) Data Acquisition Setup: The cameras are two Watec WAT-202D color cameras, operating at $25 \mathrm{~Hz}$ and connected to two Picolo PCI-bus frame grabbers. One camera is placed in front of the subject while the other was placed on the right-hand side of the subject, almost framing the object in a closeup and focussed upon it. The first camera has the view of what an external observer would be seeing of the grasp; the second would give an accurate representation of the act of grasping in full detail, including the last moments of the reaching sequence. Kinematics of the grasping act was captured using a 22-sensors Immersion CyberGlove [15] right-hand sided dataglove, which provides 22 8-bit numbers linearly related to the angles of the subject's hand joints. The resolution of the sensors is 0.5 degree. The sensors describe the position of the three phalanxes of each finger (for the thumb, rotation, and two phalanxes), the four finger-to-finger abductions, the palm arch, the wrist pitch, and the wrist yaw. The database also includes data coming from 

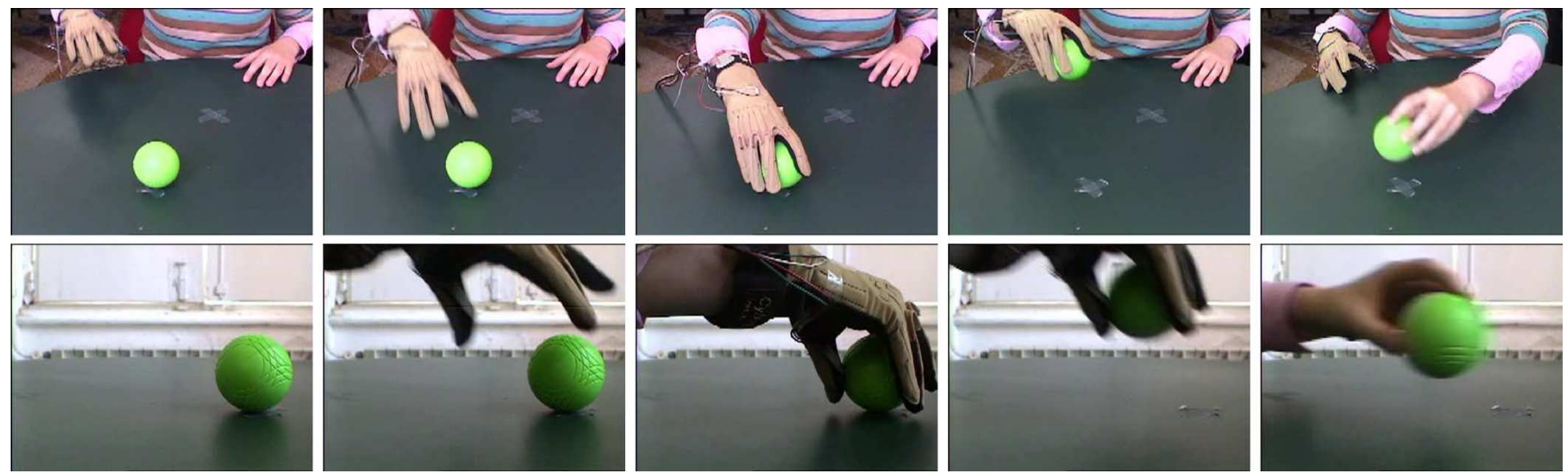

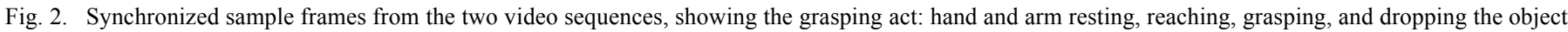
and, last, putting it back with the left arm and hand.

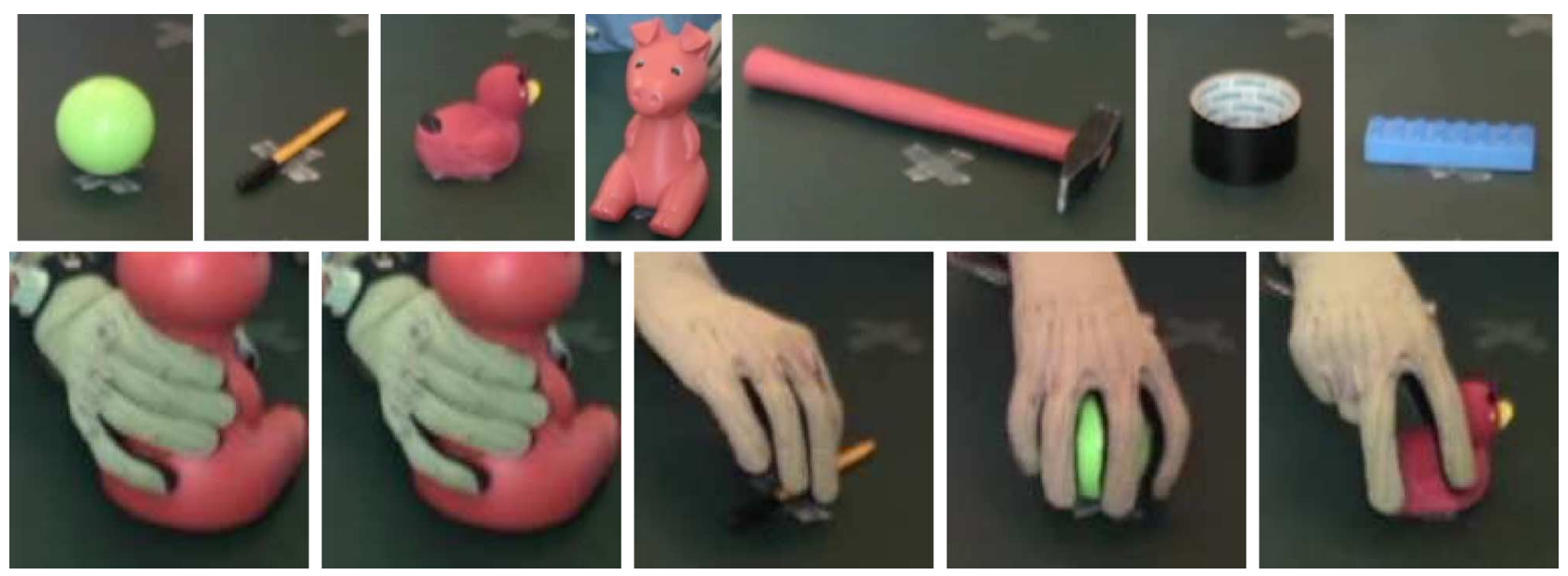

Fig. 3. Top row: the objects used in our experiments. Bottom, the grasp types we consider: (left to right) cylindric power grasp, flat grasp, pinch grip, and spherical and tripodal grip.

an Ascension Flock-Of-Birds magnetic tracker [16] mounted on the subject's wrist, which would return six real numbers, the linear and angular coordinates of the wrist with respect to a base mounted on the far end of the desk. A force sensing resistor (FSR) glued to the subject's thumb is used to determine the instant of contact with the object. All data was collected on a fast and safe mass memory storage unit and synchronized using common timestamps.

C) Objects, Subjects, and Grasps: The subjects pool includes 20 right-handed people, six females and 14 males, aged between 24 and 42 years (mean 31.5 years, median 31 ). They were engaged in grasping seven different objects in five different ways. Fig. 3 shows the objects and the grasp types. First we chose the grasps, using standard grasp taxonomies such as, e.g., Cutkosky's [4] as guidance, and trying to figure out which would be more useful in practical applications such as, e.g., dexterous teleoperation or hand prosthetics. Subsequently we chose the objects among everyday tools and toys, carefully selecting them in order for some of them to be graspable in several different ways, chosen among the grasps we had previoulsy selected. Table I sums up the total (grasp, object) pairs we have enforced (13). For instance, the pen would be grasped with either a pinch or a tripodal grip, the tape with the pinch,
TABLE I

The 13 (OBJect, Grasp) PAIRS ENForced IN THE VMGdB

\begin{tabular}{|c|c|c|c|c|c|c|c|}
\hline & ball & pen & duck & pig & hammer & tape & lego brick \\
\hline cylindr. pow. & & & & $\mathrm{X}$ & & & \\
\hline & & $\mathrm{X}$ & $\mathrm{X}$ & & $\mathrm{X}$ & $\mathrm{X}$ & $\begin{array}{l}X \\
X\end{array}$ \\
\hline spherical & $\mathrm{X}$ & & & & & $\mathrm{X}$ & \\
\hline tripodal & $\mathrm{X}$ & $X$ & $\mathrm{X}$ & & & $\mathrm{X}$ & \\
\hline
\end{tabular}

spherical or tripodal, the pig with the cylindrical power grasp, and so on.

Each subject replicated each grasp for 20 times, giving a total of $13 \times 20 \times 20=5200$ sequences, each sequence a (grasp, object, subject, $n$ ) tuple, where $n=1 \ldots 20$. (The correct number of grasping sequences was enforced by letting the subject hear the beeping sounds each time.)

\section{THEORETICAL FRAMEWORK}

We deal here with the problem of augmenting visual information about an object with motor information about it, that is the way the object can be grasped by a human being. This can be seen as an instance of a more general framework for multimodal learning. Although a formal, abstract definition of this 
TRAINING

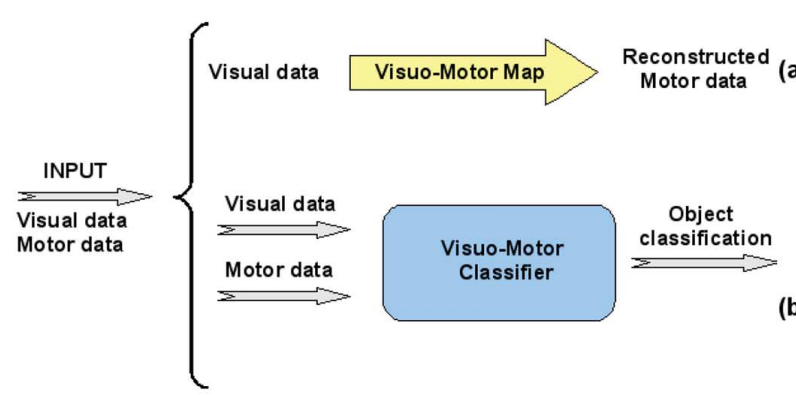

TESTING

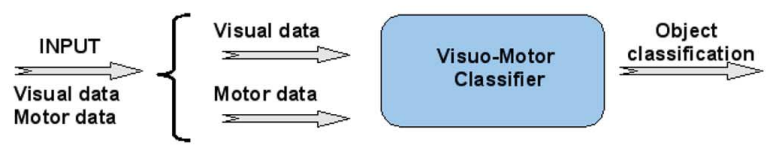

(b)

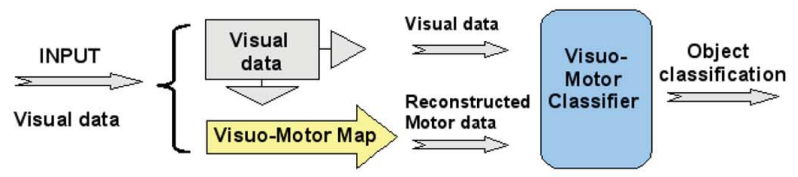

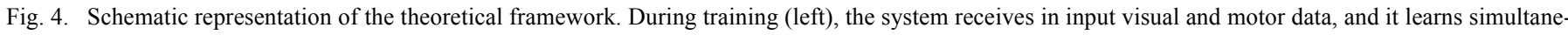

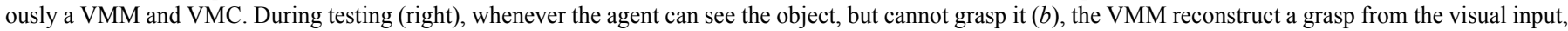
which is then used as input to the multimodal classifier jointly with the visual features.

framework is out of scope here, we outline it in order to clearly frame the point of view from which we hope to improve classical object modeling and recognition. We first give a theoretical overview of the idea, and then go in deeper detail describing the visual and motor features used, the method for training the VMM and lastly the object classifier.

\section{A. Affordances and Their Role in Object Recognition}

In everyday life, living beings use distal sensory modalities as their only means of "online" gathering information about the world (by distal here we mean, senses which operate at long distance such as, e.g., vision, hearing, smell, etc.). This is coherent with the basic needs of avoiding predators, finding food, mating and so on. Of course, (distal) sensorial information is multimodal in nature, as, e.g., the smell, sight and noise characteristic of a predator come together in experience. But to our end, a more subtle form of multimodal learning is considered, that is, associating distal and proximal modalities in the infanthood, where by proximal we mean sensorimotor and proprioceptive: those modalities which appeal to manipulation.

According to Gibson's concept of affordances, which is an instance of this general framework, the sight of an object is inextricably associated by a human being to the ways it can be used; this association is primed by manipulation in the early development: at first randomly, then in a more and more refined way. According to this, human object recognition is so good because we immediately associate to the sight of an object its affordances, and this generalizes to the case of new objects.

So, object classification should be improved by motor information, be it the real motor information or reconstructed starting from the distal modality (in this case, sight). Checking whether this idea works would in principle involve reproducing the developmental phase of manipulation in infants. Of course this is so far impossible, so we resort to building a relation between the object seen and how it can be grasped. Notice that this is in general a (nonfunctional) relation, a many-to-many relationship, since many objects can be grasped with the same grasp, and one object might afford many different grasps. In our case, to simplify the problem, we build the relation from available data gathered from adult humans, and assume that the relation is functional, meaning that it will give us only one grasp for each object it sees.
The system is then built as follows: in the training phase (see Fig. 4, left) the system is input visual and motor data, which are used to train both the VMM and the visuo-motor classifier (VMC), an object classifier which uses both visual and motor features. In the testing phase (see Fig. 4, right) the system is input either:

- visual and motor data (a); this corresponds to the case when the agent sees and grasps the object. Here the classifier receives both modalities, and it classifies the object using these informations;

- visual data only (b): this corresponds to the case when the agent sees the object but does not grasp it. In this situation, the system first reconstructs a grasp from the perceived visual features, using the VMM; then, it uses the two sets features (one perceived, one reconstructed) to classify the object.

\section{B. Implementation}

1) Visual Features: From each of the 5200 sequences, a set of relevant frames in which the object is clearly visible is extracted from the object-closeup camera stream. This is easily accomplished since the sequences are quite similar to each other in length. Background subtraction and then change detection are applied, by comparing the selected frames against a background model, in order to select a region of interest (ROI) in which the object is found. Subsequently, a bag-of-keypoints object description [17] is applied to the ROI, in order to extract from it salient visual features which can be safely associated with the object itself. Building the bag-of-keypoints description of an object is a two-phases procedure (the same idea is applied in [18], where more details can be found).

In the first phase a vocabulary of 200 visual features is built: inside each ROI a random number of points is chosen and a fixed-scale and -orientation variant of the SIFT descriptors [19] is used to characterize them. The global set of descriptors is then clustered using k-means (see, e.g., [20]) with $k=200$. This value was set after an initial round of experiments as the best found given the number of objects, sequences and characterstics of the $\mathrm{dB}$. (Notice that the optimal value of $k$ could be found automatically, e.g., using x-means [21].) The obtained 200 centroids (virtual features) are the words of the vocabulary. 
In the second phase, each object is associated to a bag of words of the vocabulary, via a simple nearest-neighbour approach. The visual appearance of the object is therefore represented by a frequency histogram of 200 bins, the $i$ th bin of the histogram indicating how many times the $i$ th word of the vocabulary is seen belonging to that object, with $i=1 \ldots 200$.

2) Motor Features: The motor features are the 22 numbers returned by the CyberGlove, considered at the time of contact of the subject's hand with the object. The value of the force-sensing resistor was used to determine the instant of contact. The motor features give a faithful snapshot of the subject's hand posture at the time of grasping the object.

3) Training the VMM: The VMM has at its core a multilayer perceptron (MLP). One single MLP in our setting has 200 input units, one hidden layer with 20 units and 22 output units; the net is trained via the scaled conjugate gradient descent method [22] and the activation is a logistic sigmoidal function. Training is done via early stopping on the appropriate validation set. (These settings are inspired by the work of Richmond and others [23], [24] on audio-to-motor mapping, probably the most similar approach to what we are presenting here.) After an initial phase of preliminary experiments, we noticed that the VMM could be largely improved by employing one MLP per each known object, and then selecting which MLP to use based upon the output of the visual classifier. If an object can be grasped in only one way (as is the case, e.g., of the hammer and pig; see Table I again), the reconstructed motor data will correspond to an estimate of this grasp; otherwise, it will represent a weighted mixture of the available grasps.

4) Training the VMC: The VMC should accept visual, motor or combined features. Algorithmically, this implies building a classifier over multiple cues. In the computer vision and pattern recognition literature some authors have suggested different methods to combine multiple cues. They can be all reconducted to one of the following three approaches: low-level, midlevel, and high-level integration [25], [26]. In the low-level case, the features are concatenated to define a single vector. In the midlevel approach, the different features descriptor are kept separated but they are integrated in a single classifier generating the final hypothesis. The high-level method starts from the output of different classifiers each dealing with one feature: the hypotheses produced are then combined together to achieve a consensus decision.

To train the VMC here we implement these three strategies in a support vector machine-based framework (SVM, see [27]). We use the discriminative accumulation scheme (DAS, see [28]) for the high level, and the multicue kernel (MCK, see [29]) for the midlevel integration. As already mentioned, the low-level integration just consists in the feature concatenation, with the new vector fed to a standard SVM. A short description of the DAS and MCK schemas follows.

DAS (High Level): DAS is based on a weak coupling method called accumulation. Its main idea is that information from different cues can be summed together. Suppose we are given $M$ object classes and for each class, a set of $N_{j}$ training data $\left\{I_{i}^{j}\right\}_{i=1}^{N_{j}}, j=1 \ldots M$. For each, we have a set of $P$ different features so that for an object $j$ we have $P$ training sets. We train an SVM on every set. Kernel functions may differ from cue to cue and model parameters can be estimated during the training step via cross validation. Given a test image $\hat{I}$ and assuming $M \geq 2$, for each single-cue SVM we compute the distance from the separating hyperplane $D_{j}(p)$, correspondent to the value of the margin obtained using the model $j$ th class versus all for cue $p$. After collecting all the distances $\left\{D_{j}(p)\right\}_{p=1}^{P}$ for all the $M$ objects and the $P$ cues, we classify the image $\hat{I}$ using the linear combination

$$
j^{*}=\underset{j=1}{\operatorname{argmax}}\left\{\sum_{p=1}^{P} a_{p} D_{j}(p)\right\}, \quad \sum_{p=1}^{P} a_{p}=1 .
$$

The coefficients $\left\{a_{p}\right\}_{p=1}^{P} \in \Re^{+}$are determined via cross validation during the training step.

MCK (Midlevel): The multicue kernel is a positively weighted linear combination of Mercer kernels, thus a Mercer kernel itself

$$
\begin{aligned}
& K_{\mathrm{MC}}\left(\left\{T_{p}\left(I_{i}\right)\right\}_{p}, \quad\left\{T_{p}(I)\right\}_{p}\right) \\
&=\sum_{p=1}^{P} a_{p} K_{p}\left(T_{p}\left(I_{i}\right), \quad T_{p}(I)\right), \quad \sum_{p=1}^{P} a_{p}=1 .
\end{aligned}
$$

In this way, it is possible to perform only one classification step, identifying the best weighting factors $a_{p} \in \Re^{+}$through cross-validation while determining the optimal separating hyperplane. This means that the coefficients $a_{p}$ are guaranteed to be optimal. Notice that cross-validation for MCK is quite time-consuming and could in principle make the obtained advantage untenable; but it is an offline preprocessing step (as well as standard cross-validation, used to find the best hyperparameters) and, once the parameters are defined, training and testing have the same computational complexity of a standard SVM. For problems in which large sample databases are available or the input space is highly dimensional, MCK can be substituted with a multikernel learning algorithm (see, for instance, [30]).

\section{EXPERIMENTAL RESULTS}

This section reports the experimental validation of our model. We begin by using real motor data (see Section IV-A), showing that by joint modeling visual and motor information it is possible to achieve a significant boost in recognition, compared to using visual information only. We proceed by evaluating the quality of the reconstructed grasp via regression (see Section IV-B). We then show that, whenever the motor information is not perceived by the agent, it is still possible to get a better performance by using the VMM (see Section IV-C).

All classification experiments are performed as follows: a training set of 130 samples and a testing set of 2600 samples (disjoint from the training set) are randomly extracted from the 5200 samples found in the VMGdB; one such training/testing set pair is called split. This procedure is repeated ten times, leading to ten splits. Every classifier is evaluated on all splits, and then the average and standard deviations of the error rate over the ten splits are reported. The error rate we use is the standard error rate for classification, i.e., the ratio of correctly predicted labels and the number of labels. This instance of crossvalidation is used to choose the best classifier hyperparameters. 


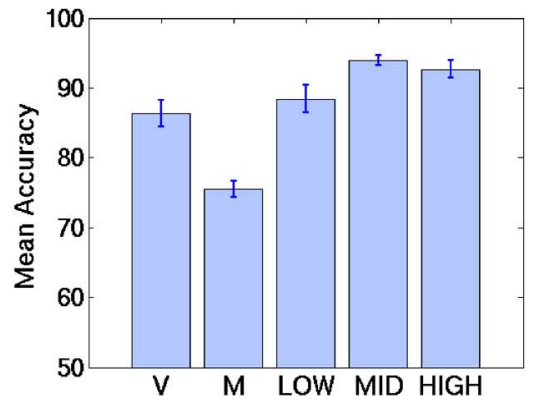

(a)

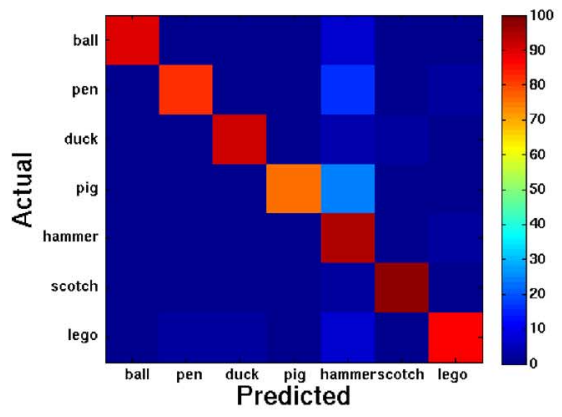

(d)

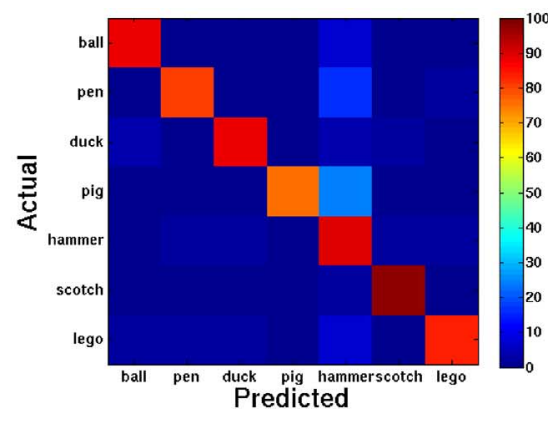

(b)

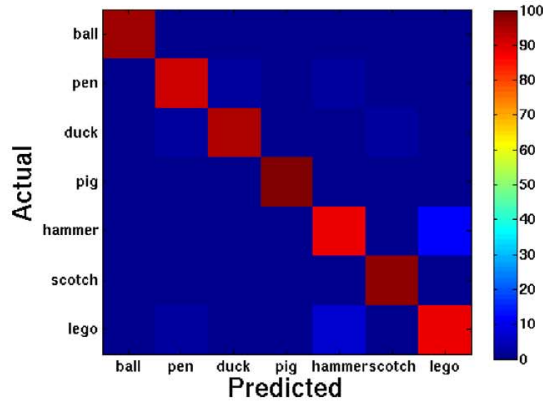

(e)

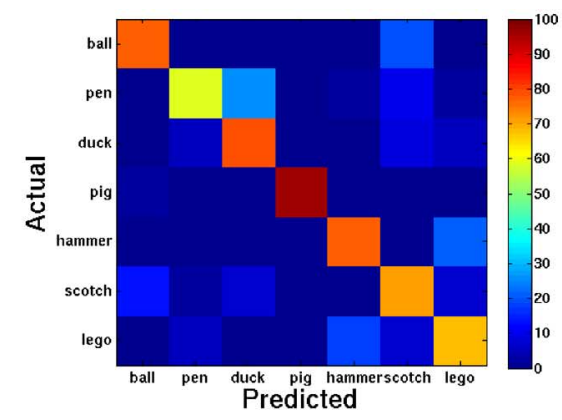

(c)

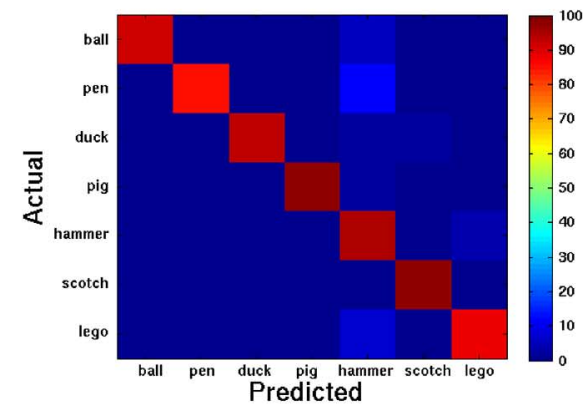

(f)

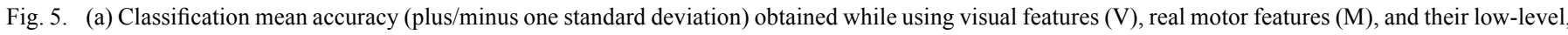
midlevel, and high-level (LOW, MID, and HIGH) integration. (b)-(f) confusion matrices using, in turn, V, M, LOW features, MIDfeatures, and HIGH features.

The classifier is a SVM, one-versus-all multiclass extension. In this extension, $N$-multiclass classification is achieved by solving $N$ two-classes classification problems, where each classifier distinguishes between one of the labels and all others; the classifier with the highest confidence determines the winning label in a winner-takes-all strategy. We use the Gaussian Kernel for the visual and motor modalities, both when considered separately and in the integration approach (two Gaussian Kernels combined in the midlevel integration schema).

\section{A. Classification With Real Motor Features}

The first set of experiments is conducted using the real motor features, namely those recorded by the users when grasping the objects, and the corresponding visual features.

Fig. 5(a) shows the overall recognition results obtained by using only visual information (V), only motor information (M), or the two combined together, with the three proposed approaches (low level, midlevel, and high level). Using the visual features a better average performance is obtained $(86.37 \% \pm 1.91 \%)$ than using the motor ones $(75.53 \% \pm 1.22 \%)$; and their integration is clearly beneficial: the midlevel integration produces the best result $(93.94 \% \pm 0.77 \%)$. The gain in accuracy between midlevel and visual only is $7.57 \%$ (difference in accuracy evaluated per split and then averaged on the ten splits). The second best result is obtained by using the high-level integration $(92.65 \% \pm 1.22 \%)$; the difference in performance between high-level and midlevel is negligible.

Fig. 5(b)-(f) shows the confusion matrices obtained by using, in turn, the visual features (b), the motor features (c), and the low-level, midlevel, and high-level integrations (d)-(f). Clearly, the combination of the two modalities leads to considerable qualitative advantages in the recognition of each object, for all methods. Consider for instance the objects "ball" and "pig": the mean accuracy is, respectively, $88.6 \%$ and $75.1 \%$ using visual features and $77.2 \%$ and $96.6 \%$ using motor features. The ball was grasped in two different ways (tripodal and spherical grasp) while the pig was manipulated only with the cylindric grasp. Thus, grasp information is object-specific for the pig, and this leads to an impressive increase in performance when using midlevel integration (100\% classification accuracy). Using integrated features is beneficial also for the ball, for which the accuracy is $96.5 \%$. Analogous considerations hold for the two other approaches. We conclude that 1) feature integration leads to a dramatic improvement in performance; and 2) the midlevel features integration is the most proficient.

\section{B. Evaluation of the VMM}

To evaluate performance of the VMM, the whole dataset was divided in a training set and a testing set, each one consisting of 2600 samples. Then:

1) the seven MLPs were trained and used to predict the motor features of the testing set;

2) a SVM was trained on the real motor features to classify the grasps, and then tested on reconstructed grasps obtained at the previous step. A predicted grasp not being one of the possible grasps associated with the related object would count as an error.

This experiment was run on ten such random (training/ testing) splits, obtaining an average error rate of $10.7 \%$, largely smaller than chance level $(63 \%)$. This indicates that the grasp reconstruction is significantly faithful to the grasps associated to the objects during the training of the VMM. 


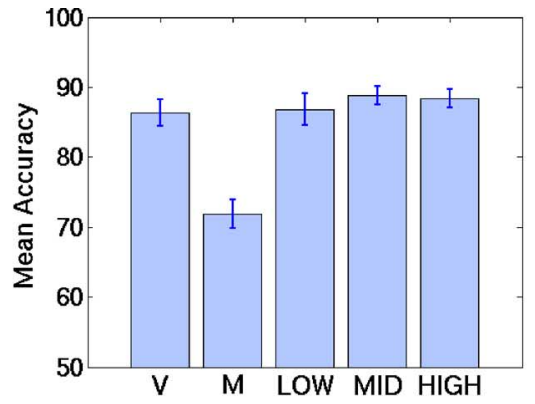

(a)

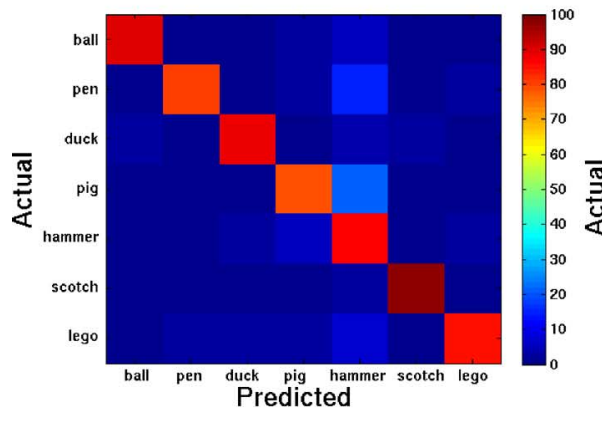

(d)

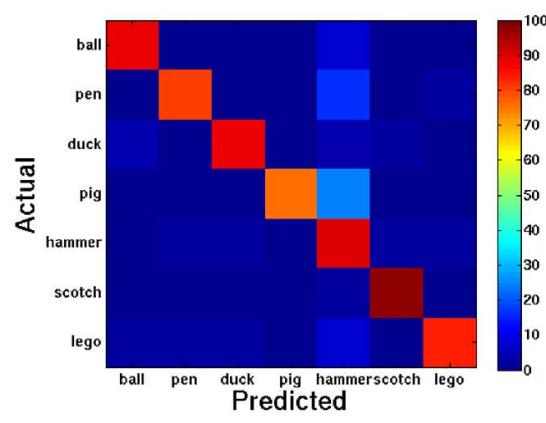

(b)

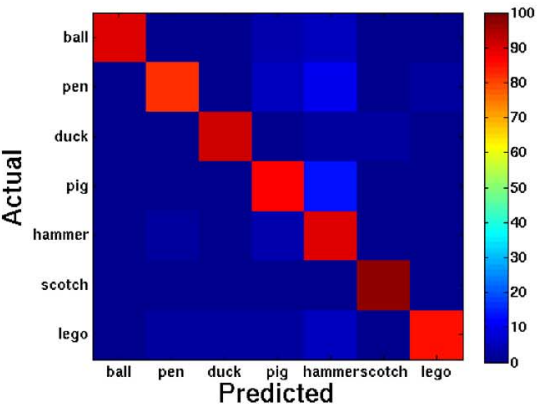

(e)

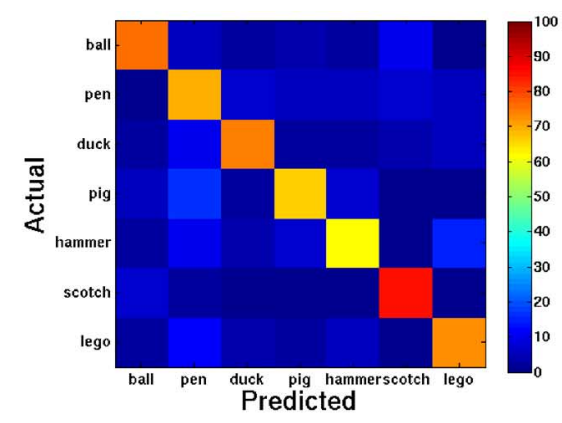

(c)

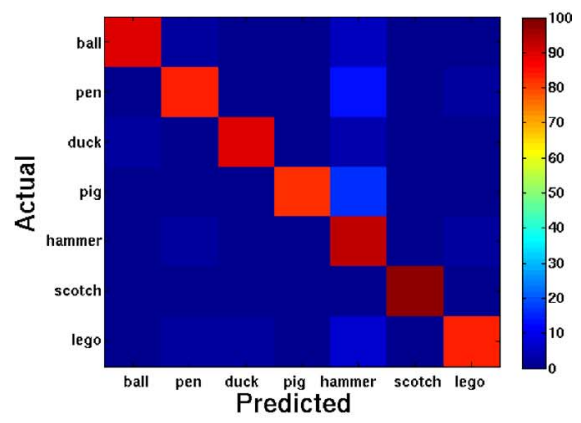

(f)

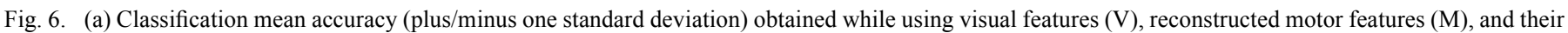
low-level, midlevel, and high-level (LOW, MID, and HIGH) integration. (b)-(f) confusion matrices using, in turn, V, M, LOW, MID, and HIGH features.

\section{Classification With Reconstructed Motor Features}

The experiments described in Section IV-A are here repeated using, instead of the real motor features, those reconstructed by the VMM. An appropriate MLP in the VMM is chosen accordingly to the prediction of the visual classifier.

Results are reported in Fig. 6. Fig. 6(a) shows the recognition rates obtained by using only visual information ( $\mathrm{V}$ - the same shown in the previous section), only motor information (M), and the two combined together (LOW, MID, HIGH). The performance of the motor only classifier decreases slightly in this case, if compared to the real features case $(71.90 \% \pm 2.06 \%$ versus $75.53 \% \pm 1.22 \%$ ). Still, the performance of the multimodal classifiers show an increase in the overall performance, compared to the vision only approach. Once again, the best performance is achieved by the midlevel integration $(88.77 \% \pm 1.29 \%)$, closely followed by the high-level $(88.38 \% \pm 1.31 \%)$.

Fig. 6(b)-(f) shows the confusion matrices obtained by all classifiers, as reported in Section IV-A. The results for the reconstructed motor data are in general lower than that obtained with the real ones [see Fig. 5(c)]. To explain this behavior there are two things to keep in mind: 1) the lower is the number of possible grasps associated with an object, the fewer are the data on which the corresponding neural network is trained; and 2) if the first step of hypothesis generation fails, the error propagates on the motor data reconstruction. In particular, both points give an intuition about why the objects "pig" and "hammer" (which were manipulated with only one grasp each) present the worst recognition results using motor information $(66.65 \%$ and $61.45 \%$, respectively). Nevertheless, in the "pig" case, the reconstructed grasp data added to the visual features brings the mean accuracy for object recognition from $75.1 \%$ (only visual) to $87.0 \%$ (using midlevel integration). As a last remark, we see once again that midlevel obtains the best performance (gain in accuracy of $2.40 \%$ ) and therefore, it appears to be the most suitable candidate for the VMC module.

\section{DISCUSSION}

The grand goal of this research is to show that the reconstruction of proximal sensory modalities can help the interpretation of the distal modalities. This very abstract idea stems from recent findings in neuroscience which seems to indicate that this is the case for a number of living beings; its theoretical value extends in principle to any distal sensory modality that one wants to understand (e.g., sound, vision). Priming and coupling of distal and proximal modalities in the infanthood is supposed to be the phase when we learn to associate, e.g., the visual appearance of a cup with the possibility of using it for drinking and carrying a liquid around.

The work hereby presented is a modest step in this direction. We focus on the problem of visual object recognition and show that the use of kinematic hand features ("motor" features) representing the ways in which the object can be grasped can significantly improve the recognition performance of a standard classifier, with respect to the case in which visual features only are employed. Actually, the experimental results indicate that motor features recorded by a sensorized glove can improve the recognition rate by some $7.6 \%$, and by $2.4 \%$ when reconstructed through the VMM. Although the latter result is not so impressive as the former, one should remember that all accuracies are here already around $90 \%$, where it is hard to gain even a few points more. Moreover, since the real motor features are so useful, 
chances are that a better VMM could improve by far the current performance of the VMM-reconstructed motor features.

In fact, the VMM is here realized via a simple regression schema based upon a MLP trained in a completely standard way; moreover, the VMM is a function whereas the relation being modeled is nonfunctional, with the result that, in some cases, its output is no physically feasible grasp, but rather a weighted average of the possible grasps. Still, it turns out to be effective, and this matches what is reported by Richmond and others in a series of papers [31], [32], [24], [33] about speech recognition; this seems to indicate that (reconstructed) motor information is really of great help, even when the reconstruction is suboptimal. Richmond's move to counter this problem was to enforce a probabilsitic model of all possible grasps rather than a function, and that is also what we plan to do as immediate future work. In that case, the VMM would be able, when seeing, e.g., the pen, to say that it would be likely grasped with a pinch or tripodal grip (consider Table I again) rather than with a power, cylindric or flat grasp. Such a VMM would enforce quite closely Gibson's concept of the affordance of the pen, somehow fully accomplishing what we said in the introduction.

Another interesting point about the VMM is that it consists of seven MLPs, hierarchically subordinated to the visual classifier; its effectiveness with respect to the simpler single MLP schema has been tested in an initial phase. How can it still be of help when the visual classifier is wrong? The answer gives more interesting insight into the problem. Let us take a step behind and consider "real" motor information. As it stands now in our framework, every object is naturally associated with the real grasp(s) it was grasped with during the experiments, and this could well be detrimental in some cases. As an example [consider again Table I and Fig. 5(b) and (e), visual features only versus midlevel integration], the visual classifier tends to confuse the pig and the hammer, due to analogies in the local descriptors, but the integration with motor features essentially eliminates the problem, since the pig is univocally associated with the cylindrical grasp and the hammer with the flat grasp. On the other hand, the motor integrated classifier shows a somehow higher confusion between the hammer and the lego brick since the lego brick too can be grasped with a flat grasp. From the motor point of view, the two objects are similar.

Let us now turn to the VMM-reconstructed features [Fig. 6(b) and (e)]. In this case, too, the pig/hammer ambiguity is resolved thanks to the motor features, and this is not surprising - it just means that the wrong MLP is input ambiguous local descriptors which still result in something close to the correct grasp for the other objects, or at least that can correct the ambiguity. On the other hand, in motor-ambiguous cases such as the hammer/lego pair, this time the VMM can correct the error since in the case of the lego it returns a weighted-average-grasp composed of the flat and pinch grasps, rather than one or the other, as it was the case with the real motor features. This mechanism is likely to explain the improvement obtained by the VMM-reconstructed features, even though the visual classifier might be wrong in the first place: it turns out that a weakness of our system, given by a simplifying assumption, is beneficial. Notice, once again, that a probabilistic description of the grasps associated to each object would solve the problem and, very likely, boost the results.

The database we presented here, the CONTACT visuo-motor drasping dataBase, (which has been using for all our experiments) is now available online for downloading (see the Note to Section I) and we hope it will represent an interesting dataset for those members of the scientific community who are willing to pursue the same path of research hereby described. The VMGdB consists of 5200 synchronized stereo video + kinematic sequences, each one representing an act of grasping. Twenty human subjects, sever objects and five grasp types are involved; ground truth is available for all sequences; and the grasping/objects relationship enforced is a many-to-many relationship.

The $\mathrm{dB}$ is as yet not comparable with other similar efforts built for pure computer vision, as far as size is concerned; but its focus is, rather than on the number of samples/objects/grasps, on the association between grasping and vision, and the variability of the subjects involved aims at giving a broad spectrum of human reaching/grasping. From this point of view, the $\mathrm{dB}$ has the potentiality to support much more research than is described here; in fact, here we have been neglecting the orientation of the hand at the time of grasping, the dynamics embedded in the reaching phase (containing a lot of information more, see, e.g., [34] and [35]) and the possibility of exploiting the two points of view (we only use one of the cameras); but these data are available in the $\mathrm{dB}$. Such a research could finally lead to a significant advance also in robotic grasping, too, as the reconstructed grasp might be somehow mapped onto the robotic end-effector in a teloperated setup.

Lastly, the approach here described is supposed to scale up to many more objects, grasp types and subjects (and in the future to the generic, online case) once the VMM is amended in the probabilistic way above described. Moreover, so far the use of fixed-scale and -orientation SIFT descriptors does not allow us to claim that this system would work in changing viewpoint conditions, but this simplifying assumption can of course be lifted. The VMGdB is collected in changing conditions of illumination, and if the local image descriptors are fully fledged SIFT, then there is a reasonable hope of robustness to illumination changes, distortion, size, and orientation changes. Future research will also be aimed at looking for better visual features.

\section{REFERENCES}

[1] J. J. Gibson, The Theory of Affordances. Hillsdale, NJ: Erlbaum, 1977.

[2] J. J. Gibson, The Ecological Approach to Visual Perception. Hillsdale, NJ: Erlbaum, 1986.

[3] B. Leibe, A. Leonardis, and B. Schiele, "Robust object detection with interleaved categorization and segmentation," Int. J. Comput. Vis., vol. 77, no. 1-3, pp. 259-289, 2008.

[4] M. R. Cutkosky, "On grasp choice, grasp models, and the design of hands for manufacturing tasks," IEEE Trans. Robot. Autom., vol. 5, no. 3, pp. 269-279, Jun. 1989.

[5] V. Gallese, L. Fadiga, L. Fogassi, and G. Rizzolatti, "Action recognition in the premotor cortex," Brain, vol. 119, pp. 593-609, 1996.

[6] G. Rizzolatti and L. Craighero, "The mirror-neuron system," Аnnu. Rev. Neurosci., vol. 27, pp. 169-192, 2004.

[7] M. Umiltà, E. Kohler, V. Gallese, L. Fogassi, L. Fadiga, C. Keysers, and G. Rizzolatti, "I know what you are doing: A neurophysiological study," Neuron, vol. 31, pp. 1-20, 2001. 
[8] J. M. Kilner, A. Neal, N. Weiskopf, K. J. Friston, and C. D. Frith, "Evidence of mirror neurons in human inferior frontal gyrus," J. Neurosci., vol. 29, pp. $10153-10159,2009$.

[9] G. Metta, G. Sandini, L. Natale, L. Craighero, and L. Fadiga, "Understanding mirror neurons: A bio-robotic approach," Interact. Stud., vol. 7, pp. 197-232, 2006.

[10] M. Lopes and J. Santos-Victor, "Visual learning by imitation with motor representations," IEEE Trans. Syst., Man, Cybern., Part B Cybern., vol. 35, no. 3, pp. 438-449, Jun. 2005.

[11] G. Griffin and P. Perona, "Learning and using taxonomies for fast visual categorization," in Proc. 21st IEEE Conf. Comput. Vis. Pattern Recognit. (CVPR), Anchorage, AK, 2008.

[12] K. Woods, D. Cook, L. Hall, K. Bowyer, and L. Stark, "Learning membership functions in a function-based object recognition system," $J$. Artif. Intell. Res., vol. 3, pp. 187-222, 1995.

[13] A. Gupta and L. Davis, "Objects in action: An approach for combining action understanding and object perception," in Proc. 21st IEEE Conf. Comput. Vis. Pattern Recognit. (CVPR), Anchorage, AK, 2008.

[14] H. Kyellstrom, J. Romero, D. Martinez, and D. Kragic, "Simultaneous visual recognition of manipulation actions and manipulated objects," in Proc Eur. Conf. Comput. Vis. (ECCV), Marseille, France, 2008.

[15] CyberGlove Reference Manual. Palo Alto, CA: Virtual Technologies, Inc., Aug. 1998

[16] The Flock of Birds - Installation and Operation Guide. Burlington, VT: Ascension Technology Corporation, Jan. 1999.

[17] G. Csurka, C. R. Dance, L. Fan, J. Willamowski, and C. Bray, "Visual categorization with bags of keypoints," in Proc. Workshop Stat. Learn. Comput. Vis., ECCV, Prague, Czech Republic, 2004, pp. 1-22.

[18] N. Noceti, B. Caputo, C. Castellini, L. Baldassarre, A. Barla, L. Rosasco, F. Odone, and G. Sandini, "Towards a theoretical framework for learning multi-modal patterns for embodied agents," in Proc. 15th Int. Conf. Image Anal. Process. (ICIAP), Solerno, Italy, 2009.

[19] D. Lowe, "Distinctive image features from scale-invariant keypoints," Int. J. Comput. Vis., vol. 60, no. 2, pp. 91-110, 2004.

[20] T. Cover and P. Hart, "Nearest neighbor pattern classification," IEEE Trans. Inf. Theory, vol. 13, no. 1, pp. 21-27, Jan. 1967.

[21] D. Pelleg and A. Moore, "X-means: Extending k-means with efficient estimation of the number of clusters," in Proc. 17th Int. Conf. Mach. Learn., Stanford, CA, 2000, pp. 727-734.

[22] M. F. Moller, "A scaled conjugate gradient algorithm for fast supervised learning," Neural Netw., vol. 6, pp. 525-533, 1993.

[23] G. Papcun, J. Hochberg, T. R. Thomas, F. Laroche, J. Zacks, and S. Levy, "Inferring articulation and recognizing gestures from acoustics with a neural network trained on X-ray microbeam data," J. Acoust. Soc. Amer., vol. 92, no. 2, 1992.

[24] K. Richmond, "Trajectory mixture density networks with multiple mixtures for acoustic-articulatory inversion," in Proc. Int. Conf. Adv. Nonlinear Speech Process., M. Chetouani, A. Hussain, B. Gas, M. Milgram, and J.-L. Zarader, Eds., Dec. 2007, vol. 4885, pp. 263-272, Springer-Verlag Berlin Germany, ser. Lecture Notes in Computer Science.

[25] R. Polikar, "Ensemble based systems in decision making.," IEEE Circuits Syst. Mag., vol. 6, no. 3, pp. 21-45, Sep. 2006.

[26] C. Sanderson and K. K. Paliwal, "Identity verification using speech and face information," Digital Signal Process., vol. 14, no. 5, pp. 449-480, 2004.

[27] B. E. Boser, I. M. Guyon, and V. N. Vapnik, "A training algorithm for optimal margin classifiers," in Proc. 5th Annu. ACM Workshop Comput. Learn. Theory (COLT), D. Haussler, Ed., Pittsburgh, PA, 1992, pp. 144-152, ACM press.

[28] M. Nilsback and B. Caputo, "Cue integration through discriminative accumulation," in Proc. IEEE Computer Soc. Conf. Comput. Vis. Pattern Recogn., Washington, DC, 2004, vol. 2, pp. 578-585.

[29] T. Tommasi, F. Orabona, and B. Caputo, "Discriminative cue integration for medical image annotation," Pattern Recognit. Lett., vol. 29, no. 15 , pp. 1996-2002, 2008.

[30] F. Orabona, L. Jie, and B. Caputo, "Online-batch strongly convex multi kernel learning," in Proc. 23rd IEEE Conf. Comput. Vis. Pattern Recognit. (CVPR), San Francisco, CA, 2010.

[31] K. Richmond, S. King, and P. Taylor, "Modelling the uncertainty in recovering articulation from acoustics," Comput. Speech Lang., vol. 17, no. 2, pp. 153-172, 2003.

[32] A. A. Wrench and K. Richmond, "Continuous speech recognition using articulatory data," in Proc. Int. Conf. Spoken Lang. Process., Beijing, China, 2000.
[33] S. King, J. Frankel, K. Livescu, E. McDermott, K. Richmond, and M. Wester, "Speech production knowledge in automatic speech recognition," J. Acoust. Soc. Amer., vol. 121, no. 2, pp. 723-742, 2007.

[34] C. Bard, J. Troccaz, and G. Vercelli, "Shape analysis and hand preshaping for grasping," in Proc. IEEE/RSJ Int. Workshop Intell. Robots Syst., Tokyo, Japan, Nov. 1991, vol. 1, pp. 64-69.

[35] S. A. Winges, D. J. Weber, and M. Santello, "The role of vision on hand preshaping during reach to grasp," Exp. Brain Res., vol. 153, pp. 489-498, 2003.

C. Castellini received the doctorate degree in artificial intelligence from the University of Edinburgh, Edinburgh, U.K., in 2005.

He then spent 4.5 years as a postdocoral researcher in the Advanced Robotics Laboratory of the University of Genova, Genova, Italy, working on machine learning applied to human sensorimotor data. He is currently a researcher at the DLR, German Aerospace Center. He has coauthor of more than 25 peerreviewed papers.

T. Tommasi received the M.Sc. degree in physics and the Dipl. degree in medical physics specialization from the University of Rome, La Sapienza, Italy, in 2004 and 2008, respectively. She is currently working towards the Ph.D. degree in electrical engineering at the École Polytechnique Fédérale de Lausanne, Lausanne, Switzerland.

She is currently a Research Assistant at the Idiap Research Institute, Martigny, Switzerland. Her research interests include machine learning and computer vision with a particular focus on knowledge transfer and object categorization using multimodal information.

N. Noceti received the B.Sc. and Ph.D. degrees in computer science from the Universit di Genova, Genova, Italy, in 2006 in 2010, respectively.

She is currently a Postdoctoral Researcher at DISI, where she works within the SlipGURU group. Her research interests include video analysis and its application to video surveillance. She is interested in the study, the design and the development of computer vision techniques combined with statistical learning from examples for action and event analysis, behavior modeling, and scene understanding.

F. Odone received the Laurea degree in information sciences and the Ph.D. degree in computer science from the University of Genova, Genova, Switzerland, in 1997 and 2002, respectively.

She visited Heriot-Watt University, Edinburgh, U.K., in 1997 as a research associate and as a visiting Ph.D. student in 1999. From 2002 to 2005, she was a researcher at INFM (CNR). Since the end of 2005, she has been an Assistant Professor in the Department of Computer and Information Sciences of the University of Genova, Genova, Italy. She published over 50 papers on international journals and conference proceedings covering many aspects of computer vision and machine learning. Her current research interests include theory and algorithms for classification, variable selection, unsupervised or semi-supevised knowledge extraction from high dimensional sets; object detection and recognition methods for images and image sequences; behavior modeling and classification; design and development of applications and prototypes for videosurveillance and biometry; and the study and development of computer vision methods for extracting 3-D information on the observed scene.

B. Caputo received the Ph.D. degree in computer science from the Royal Institute of Technology (KTH), Stockholm, Sweden, in 2004.

Since 2006, she has been a Senior Research Scientist at the Idiap Research Institute, Martigny, Switzerland, where she leads the Cognitive Vision Systems group. She previously studied physics and biomedical signal processing at the University of Rome, La Sapienza, Italy, where she won the award for the best Master Thesis in Biomedical Signal Processing in 2000. Her main research interests include developing algorithms for learning, recognition, and categorization for artificial autonomous systems like robots. 